\title{
Renal-related adverse effects of intravenous contrast media in computed tomography
}

\author{
Kheng Song $\underline{L e o w}^{1}$, MD, Yi Wei $\underline{W u}^{1}$, MD, Cher Heng $\underline{T a n}^{1}$, MBBS, FRCR
}

\begin{abstract}
Renal-related adverse effects of intravascular contrast media (CM) include contrast-induced nephropathy in computed tomography and angiography. While large retrospective studies have been published, the exact pathogenesis of this condition is still unknown. We review the main international guidelines, including the American College of Radiology white paper and the guidelines of European Society of Urogenital Radiology, Royal College of Radiologists and Canadian Association of Radiologists, as well as their references, regarding this subject. We present a simplified, concise approach to renal-related adverse effects of $\mathrm{CM}$, taking into consideration the basis for each recommendation in these published guidelines. This will allow the reader to better understand the rationale behind appropriate patient preparation for crosssectional imaging.
\end{abstract}

Keywords: contrast, iodinated, nephrogenic systemic fibrosis, nephropathy

\section{INTRODUCTION}

In 1978, the first nonionic intravenous contrast medium (CM), i.e. metrimazide (Amipaque), was approved in the United States. Since then, the diagnostic capability of physicians, especially those in the fields of urology and cardiology, has been immensely revolutionised. ${ }^{(1)}$ Following the increased use of intravenous iodinated CM, a higher number of CM-related adverse effects has been recognised, with contrast-induced nephropathy $(\mathrm{CIN})$ constituting one of the most serious adverse effects. ${ }^{(2)}$

$\mathrm{CIN}$ is also known as contrast-induced acute kidney injury. ${ }^{(3)}$ Nephrology literature on acute kidney injury have proposed several definitions and classifications for CIN. ${ }^{(4,5)}$ However, the most widely quoted definition is from the Contrast Media Safety Committee of the European Society of Urogenital Radiology (CMSC ESUR), in which CIN is defined as a deterioration of renal function (defined as an increase in serum creatinine by more than $25 \%$ or $44 \mu \mathrm{mol} / \mathrm{L}$ ) within three days of intravascular administration of $\mathrm{CM}$ in the absence of an alternative aetiology ${ }^{(6)}$

$\mathrm{CIN}$ has been reported as the third most common cause of hospital-acquired renal failure, with an incidence rate of $11 \%$. $^{(7)}$ A cohort study in a university hospital cardiac centre in Singapore showed a CIN incidence rate of up to $11.4 \%$ following intraarterial administration under percutaneous coronary intervention $(\mathrm{PCl}){ }^{(8)} \mathrm{CIN}$ is also associated with an increased mortality rate of $9.7 \% .{ }^{(9)}$ Besides prolonging hospital stay, ${ }^{(10)} \mathrm{CIN}$ was shown to increase the incidence of a range of cardiovascular events, from coronary disease to stroke. ${ }^{(11)}$

With the rapidly ageing population and the increased prevalence of chronic kidney diseases (CKD), radiologists and referring physicians ought to be familiar with up-to-date and evidence-based practices of CIN. Each of the main international guidelines elaborates on different aspects of $\mathrm{CIN}$ management and at varying depths. Some guidelines adopt a slightly different approach from others, e.g. the management of metformin in patients with diabetes mellitus. In view of the complementary roles of international guidelines, this article aims to review and summarise the four main international guidelines: CMSC ESUR guidelines version 8.1; American College of Radiology (ACR) Manual on Contrast Media version 9 2013; Consensus Guidelines for the Prevention of Contrast Induced Nephropathy by Canadian Association of Radiologists (CAR) 2011; and Standards for Intravascular Contrast Agent Administration to Adult Patients by the Royal College of Radiologists (RCR) second edition 2010. The references within the aforementioned guidelines are also examined, with the related articles retrieved from the PubMed database.

\section{PATHOPHYSIOLOGY OF CIN}

All CM are rapidly distributed into intravascular and extracellular fluids following intravascular administration. They are solely eliminated by glomerular filtration. ${ }^{(7)}$ Extrarenal excretion constitutes less than $1 \%$ in normal renal function. ${ }^{(12)}$ It has been proven that approximately $100 \%$ of CM is excreted within the first 24 hours after administration in patients with normal renal function. ${ }^{(7)}$ On the contrary, in patients with reduced renal function, the half-life of elimination can increase by up to 40 hours or more. ${ }^{(7)}$

Under physiological resting condition, $25 \%$ of the cardiac output is directed to the kidneys. ${ }^{(7)}$ The majority of vascular flow is channelled toward the cortex to optimise glomerular filtration and reabsorption of water and salts. ${ }^{(7)}$ Renal medullary blood flow is, on the contrary, low. ${ }^{(7,13)}$ This makes the renal medulla prone to ischaemic injury, cellular damage and consequently, CIN. ${ }^{(14)}$ The exact pathophysiology of $\mathrm{CIN}$ is not well understood. The accepted aetiologic factors comprise three different but potentially interacting pathways: the haemodynamic effects of $\mathrm{CM}$; the effects of reactive oxygen species (ROS); and direct tubular cellular toxicity by $\mathrm{CM}$ molecules. ${ }^{(7,15)}$ 


\section{Haemodynamic effects}

The deeper portion of the outer medulla of the kidney is metabolically active; hence, it is predisposed to hypoxic injury. ${ }^{(7)}$ Following administration of CM, a biphasic haemodynamic response occurs - a brief initial phase of increased renal blood flow, followed by prolonged flow reduction by $10 \%-25 \%$ below the baseline. This decreases the partial pressure of oxygen $\left(\mathrm{PO}_{2}\right.$ ) of the outer medulla by $50 \%-67 \%$ to the level of 9-15 mmHg (compared to $20 \mathrm{mmHg}$ under physiological condition). Furthermore, the higher the osmolality of the $\mathrm{CM}$, the higher the oxygen requirement of the tubular cells, making them even more vulnerable to hypoxic injury and $\mathrm{CIN} .{ }^{(14)}$

\section{Effects of ROS}

Medullary hypoxia following CM administration also leads to an increased formation of ROS. ${ }^{(7)}$ These ROS include superoxide $\left(\mathrm{O}_{2}^{-}\right)$, hydroxyl radicals $\left(\mathrm{OH}^{-}\right)$and less aggressive reacting molecules such as hydrogen peroxide $\left(\mathrm{H}_{2} \mathrm{O}_{2}\right){ }^{(15,16)}$ Once exceeding the scavenging capabilities of antioxidants, these ROS cause oxidative stress and lead to ischaemia reperfusion injury at the cellular level. ${ }^{(7)}$ ROS also triggers and increases angiotensin II- and endothelin I-induced vasoconstriction, and decreases the bioavailabilty of vasodilative nitric oxide (NO), thus compromising the ischaemic state of the outer medulla. This whole process forms a vicious cycle, leading to $\mathrm{CIN} .{ }^{(17)}$

\section{Effects of tubular cell toxicity}

Other effects of CM on tubular cells include intercellular junction disruption, membrane protein redistribution, DNA fragmentation, reduction of extracellular $\mathrm{Ca}^{2+}$, and even altered mitochondrial function. ${ }^{(18)}$ High osmolality CM (HOCM) has been shown to produce a more pronounced toxic effect than low- or iso-osmolar $\mathrm{CM}^{(7)}$ but HOCM is fortunately not used in current practice. Historically, sodium acetrizoate (Urokon $\left.{ }^{\circledR}\right)$ was the first ionic HOCM that was synthesised in 1953, followed by sodium diatrizoate (Urografin ${ }^{\circledR}$ ) and sodium iothalamate (Conray $\left.{ }^{\circledR}\right)$. Due to the serious adverse effects from the high osmolality and ionicity, HOCM have been replaced by low osmolality CM (LOCM).

In summary, CIN occurs as a result of medullary ischaemia following both increased oxygen consumption by tubular cells and decreased perfusion in the renal outer medulla. These involve interacting processes of ROS formation, imbalance between vasoconstrictive and vasodilatative mediators, and direct toxicity on the tubular cells. ${ }^{(7)}$

\section{MAIN PREDICTOR OF CIN - RENAL IMPAIRMENT}

The most important predictor of $\mathrm{CIN}$ is renal impairment. ${ }^{(3)}$ Renal impairment increases the risk of CIN by more than 20 times. ${ }^{(19,20)}$ In patients with normal renal function, the development of $\mathrm{CIN}$ after intravascular CM is extraordinarily rare, or does not occur at all. ${ }^{(3,19)}$ With declining renal function, there is a corresponding increased risk of CIN. ${ }^{(6)}$ Numerous studies have shown that the incidence of $\mathrm{CIN}$ varies from less than $2 \%$ in the general population to up to $50 \%$ in patients with advanced renal diseases. ${ }^{(7)}$

Conventionally, in patients receiving intra-arterial injection, a cautious threshold of estimated glomerular filtration rate (eGFR) $<60 \mathrm{~mL} / \mathrm{min}$ is practised.(6) This route of CM administration is more commonly seen in cardiovascular angiography and vascular interventional radiology. Consensus guidelines from CAR classify this category of patients as moderate to high risk. This is because intra-arterial injection is associated with at least twice the risk of $\mathrm{CIN}$ and poorer outcomes compared to the intravenous route of administration. ${ }^{(3)}$ This practice is supported by numerous radiology literature; for instance, Moore et al's study involves a randomised double-blinded clinical trial comparing between groups of patients who underwent angiocardiography and computed tomography $(\mathrm{CT})$, which showed a relative odds of 3.44. ${ }^{(21)}$

One recent major change to eGFR cutoffs is outlined in the updated guidelines from CMSC ESUR. ${ }^{(6)}$ For patients receiving intravenous $C M$, the precautionary cutoff level has been lowered from eGFR $<60 \mathrm{~mL} / \mathrm{min}$ to eGFR $<45 \mathrm{~mL} / \mathrm{min}$. This is because data and review of intravenous CM administration studies have shown that the risk of CIN increases only when eGFR is $<45 \mathrm{~mL} / \mathrm{min}$. This is further supported by a study by Katzberg and Barrett in 2007, which showed that the incidence of CIN in patients with eGFR $<45 \mathrm{~mL} / \mathrm{min}$ varied between $5 \%$ and $20 \%$ as compared to less than $1 \%$ in those with eGFR $>45 \mathrm{~mL} / \mathrm{min} .{ }^{(22)}$

Manuals from ACR and guidelines from RCR have yet to discriminate between intra-arterial and intravenous routes of administration for CIN risk assessment. ${ }^{(19,23)}$ However, ACR manuals have mentioned that the higher overall incidence of $\mathrm{CIN}$ reported in several publications ${ }^{(24)}$ is likely an overestimation among patients undergoing intravenous contrast-enhanced studies. ${ }^{(19)}$ RCR guidelines state that the eGFR level chosen to trigger special precautions may be set locally after discussion between local radiologists and nephrologists. ${ }^{(23)}$

\section{OTHER RISK FACTORS FOR CIN}

Apart from renal impairment, comorbidities are equally important risk factors for $\mathrm{CIN}$, as the risk and severity of $\mathrm{CIN}$ increases proportionally with the number and severity of risk factors. ${ }^{(6)}$ In 2004, Mehran et al ${ }^{(25)}$ introduced a simple scoring system for the prediction of $\mathrm{CIN}$ risk after $\mathrm{PCl}$, which involves eight risk factors, as shown in Fig. 1. Each of these risk factors is assigned an integer score, and the scores are then summed up to calculate both CIN risk and risk requiring dialysis. ${ }^{(25)}$

ESUR guidelines categorise risk factors for CIN as patient- or procedure-related. ${ }^{(6)}$ Patient-related risks encompass diabetic nephropathy, dehydration, congestive cardiac failure (New York Heart Association [NYHA] II-IV), low left ventricular ejection fraction, gout, age $>70$ years, concurrent administration of nephrotoxic drugs, e.g. non-steroidal anti-inflammatory drugs (NSAIDs), and known or suspected acute renal failure. The list of patient-related risk factors has been expanded to include factors related to cardiovascular instability, such as periprocedural hypotension, recent myocardial infarction $(<24$ hours), low 


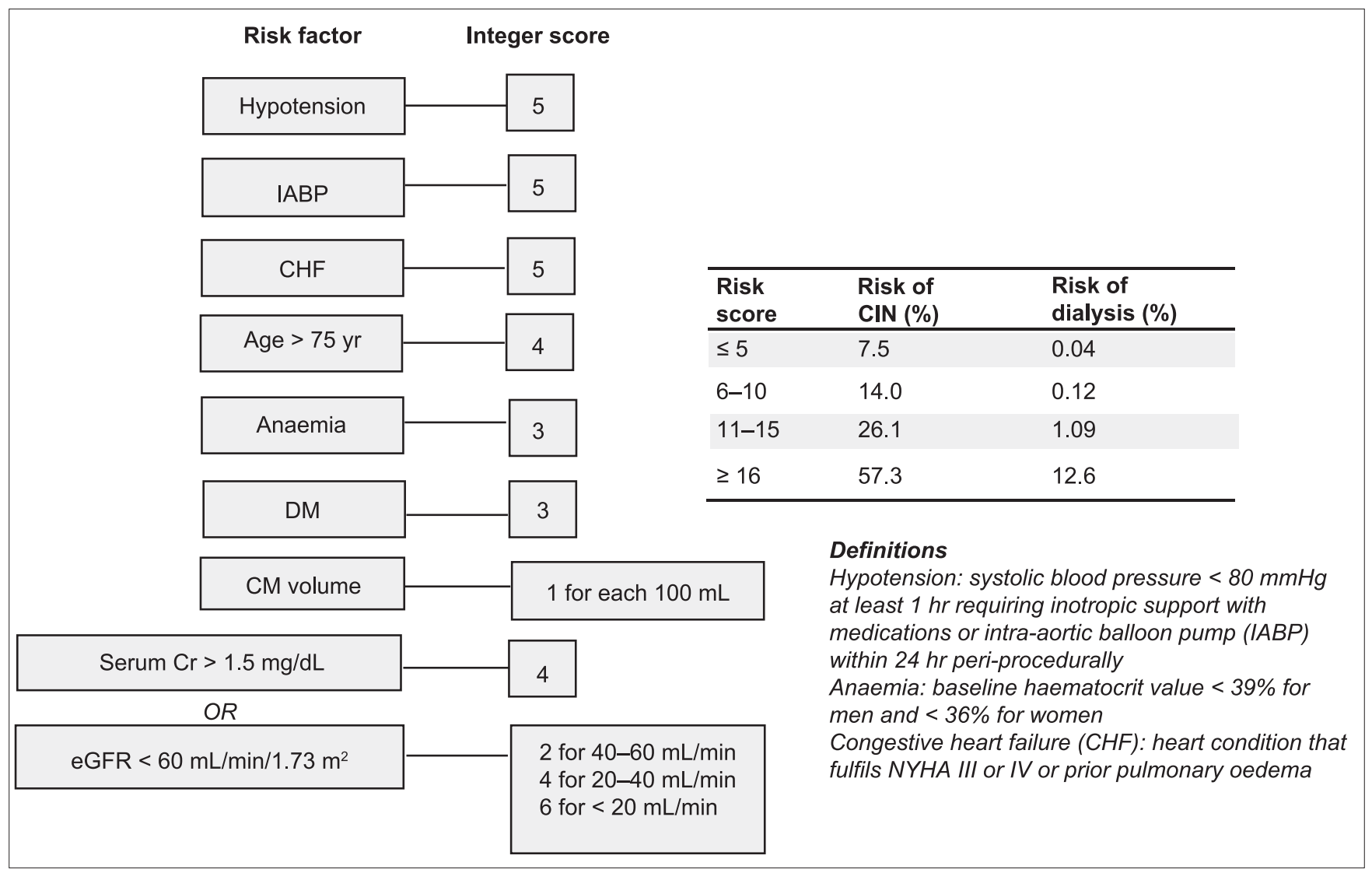

Fig. 1 Mehran's scoring system. ${ }^{(25)} \mathrm{CIN}$ : contrast-induced nephropathy; CM: contrast media; Cr: creatinine; DM: diabetes mellitus; eGFR: estimated glomerular filtration rate; NYHA: New York Heart Association

haematocrit level and use of an intra-aortic balloon pump. Procedure-related risks are related to intra-arterial route of administration, high osmolality agents, large dosage and multiple administrations within an interval of a few days. ${ }^{(6)}$ These risk factors are summarised in Table I.

Consensus from CAR includes the following as part of the contributing factors of $\mathrm{CIN}$ : sepsis; previous chemotherapy; organ transplants; human immunodeficiency virus; and collagen vascular diseases. ${ }^{(3)}$ In addition, ACR manuals incorporate hyperuricaemia as one of the risk factors for CIN. ${ }^{(19)}$ Studies by Okino et $\mathrm{al}^{(26)}$ in 2010 and Saritemur et $\mathrm{al}^{(27)}$ in 2013 demonstrated hyperuricaemia as an early predictor of slow and mild development of renal insufficiency after $\mathrm{PCl}$.

\section{SCREENING OF SERUM CREATININE BEFORE INTRAVASCULAR CM ADMINISTRATION: IS IT NECESSARY?}

Routine measurement of serum creatinine and eGFR is not practical, and may delay scheduled investigations, disrupt bookings and incur extra costs. ${ }^{(28,29)}$ The validity of an available serum creatinine level for $\mathrm{CIN}$ risk assessment can range from one week to six months, depending on the pre-existing guidelines or manuals. ${ }^{(3,6,19,23)}$

CAR consensus suggests the measurement of serum creatinine and eGFR within six months in stable outpatients with one or more risk factors but without significant renal impairment. ${ }^{(3)}$ More recent serum creatinine measurement (within an interval of
Table I. Risk factors for contrast-induced nephropathy in intravenous iodinated contrast agents based on the European Society of Urogenital Radiology guidelines. ${ }^{(6)}$

\section{Patient-related risk factors:}

a) Renal impairment is the most important predictor

- eGFR $<60 \mathrm{~mL} / \mathrm{min} / 1.73 \mathrm{~m}^{2}$ before intra-arterial administration

- eGFR $<45 \mathrm{~mL} / \mathrm{min} / 1.73 \mathrm{~m}^{2}$ before intravenous administration

b) Other risk factors include:

- Diabetic nephropathy

- CHF (NYHA III-IV) and low LV ejection fraction

- Dehydration

- Age $>70$ yr

- Anaemia

- Concurrent use of nephrotoxic drugs, e.g. NSAIDS

- Known or suspected acute kidney injury

c) Cardiovascular instability includes:

- Peri-procedure hypotension

- Recent myocardial infarction $<24 \mathrm{hr}$

- Low haematocrit

- Use of intra-aortic balloon pump

2. Procedure-related risk factors:

- Intra-arterial administration

- High osmolality agents

- Large doses of contrast media

- Multiple administrations within a few days' interval

CHF: congestive heart failure; eGFR: estimated glomerular filtration rate; LV: left ventricular; NSAIDS: nonsteroidal anti-inflammatory drugs; NYHA: New York Heart Association

one week) is needed for inpatients and patients with unstable or acute renal disease. ${ }^{(3)}$ RCR guidelines corroborate with the CAR consensus, but recommends a shorter duration of three months 
for patients with stable clinical condition. A 'recent' measurement is recommended for diabetic patients or patients requiring intraarterial injection, although no specific interval was outlined. A risk-versus-benefit approach has to be applied in acutely or severely unwell patients, such as those with hypotension or hypovolaemia. ${ }^{(23)}$

According to the ESUR guidelines, serum creatinine (and eGFR) measurement within one week's interval is required for patients with known eGFR $<60 \mathrm{~mL} / \mathrm{min} / 1.73 \mathrm{~m}^{2}$, patients who require intra-arterial $\mathrm{CM}$, patients aged $>70$ years, patients with a history of diabetes mellitus, hypertension (not necessarily requiring medical therapy as opposed to ACR guidelines), gout, renal disease, renal surgery, proteinuria, or who were on recent nephrotoxic drugs. ${ }^{(6)}$ The ACR manuals state that there is no universally accepted interval between the baseline serum creatinine measurement and CM administration. Some have accepted a 30-day interval as adequate, with a shorter interval for inpatients and those with new risk factors for renal dysfunction. ${ }^{(19)}$

In routine clinical practice, we frequently encounter outpatients who present for contrast-enhanced studies without a baseline serum creatinine level. CIN risk assessment is therefore limited, and options include an alternative noncontrast study or on-the-spot serum creatinine measurement. This has resulted in delayed clinic appointment, patient dissatisfaction, extra administrative work, greater time consumption, and even compromised radiological analysis if plain cross-sectional imaging is opted. ${ }^{(30)}$ Choyke et $\mathrm{al}^{(30)}$ supplemented this gap in their study, which found that patients who had abnormal serum creatinine level with high specificity could be excluded from serum testing prior to contrast injection for imaging studies. Patients in the study completed a questionnaire, which included questions on the presence of the following risk factors: preexisting renal dysfunction; proteinuria; prior renal surgery; hypertension; diabetes mellitus; and gout. The study suggested that if all six of these survey questions were answered in the negative, $94 \%$ of patients would have a normal serum creatinine level and 99\% would have a serum creatinine level under $1.7 \mathrm{mg} / \mathrm{dL}(150.3 \mu \mathrm{mol} / \mathrm{L})$. Thus, the authors concluded that patients without the aforementioned risk factors could reasonably be excluded from serum creatinine screening prior to contrast injection. ${ }^{(30)}$

It is worth emphasising that serum creatinine is not a reliable indicator of renal function, as the normal serum creatinine level is usually maintained until the GFR is reduced by nearly $50 \% .{ }^{(19)}$ According to the Modification of Diet in Renal Disease (MDRD) or Cockcroft-Gault formulae, eGFR in adults is widely accepted as an index of renal function. ${ }^{(19)}$ However, one should bear in mind that the MDRD formula is known to underestimate eGFR in patients with normal or nearnormal renal function. ${ }^{(31)}$ The formula is intended for use in patients with CKD. It is not designed to monitor acute changes in renal function, and therefore, does not perform well in ill, hospitalised patients, who make up the majority that require radiological imaging. ${ }^{(32)}$

\section{METFORMIN AND INTRAVASCULAR CM USE}

With a rising trend of metformin use among patients with diabetes mellitus, radiologists and clinicians should be well versed with metformin management during the period of $\mathrm{CM}$ administration. Approximately $90 \%$ of the administered metformin is eliminated via the kidneys in 24 hours. ${ }^{(12)}$ Metformin itself does not confer an increased risk of CIN. ${ }^{(19)}$ Instead, it carries a very rare risk of lactic acidosis in patients with renal failure. ${ }^{(19)}$

\section{PREVENTIVE MEASURES OF CIN}

$\mathrm{CIN}$ usually manifests as transient asymptomatic elevation of serum creatinine, which begins within 24 hours of contrast injection, peaks within 4 days, and returns to baseline within 7-10 days. ${ }^{(33)}$ It is not commonly associated with permanent renal dysfunction. ${ }^{(19)}$ Despite a self-limiting clinical course in most cases, ${ }^{(6)}$ prevention is crucial to avoid increased morbidity and mortality. ${ }^{(7)}$ The majority of the international guidelines practise the same preventive measures in reducing the risk of CIN. These will be discussed in-depth in the relevant sections and systematically outlined as 'before', 'during' and 'after' the procedure.

\section{Before the procedure}

The first step to preventing CIN is to identify at-risk patients. These patients should be considered for alternative imaging modalities that do not require $\mathrm{CM}$ injection, such as ultrasonography or magnetic resonance (MR) imaging. There is no universally agreed upon threshold of serum creatinine elevation that contraindicates the administration of CM. ACR manuals state that there is insufficient data at this time to prescribe a specific recommended threshold. ${ }^{(19)}$ However, it is believed that the risk of $\mathrm{CIN}$ is sufficiently low for eGFR $>45 \mathrm{~mL} / \mathrm{min}$ and is likely to be safe for most patients. ${ }^{(22)}$ The bottomline practice lies in weighing the risks and benefits. ${ }^{(23)}$ There may also be a need to discuss with the referring physician to stop any nephrotoxic drugs, e.g. stop NSAIDs for at least 24-48 hours. ${ }^{(6)}$

Fluid volume expansion and avoidance of dehydration are the mainstays of CIN prevention. The principle of volume expansion is to increase intravascular volume, renal blood flow and diuresis, reduce the contact time of $\mathrm{CM}$ with renal tubular cells, and suppress the renin-aldosterone system. ${ }^{(7)}$ Controversy exists in the types and routes of hydration, as well as the optimal duration required. A simplified approach to $\mathrm{CIN}$ prevention is outlined in Table II.

\section{Type of fluid regime: $0.9 \%$ vs. $0.45 \%$ of normal saline}

Both the ESUR and ACR guidelines unanimously agree that $0.9 \%$ saline is superior to $0.45 \%$ saline in the reported risk of CIN reduction. ${ }^{(6,19)}$ This is supported by studies conducted by Weishord et al and Mueller et al in the year 2008 and 2002, respectively. ${ }^{(34,35)}$ However, the infusion rate varies according to different guidelines. ESUR guidelines suggest an infusion rate of $1.0-1.5 \mathrm{~mL} / \mathrm{kg} / \mathrm{hr}$ for at least 6 hours before and 6 hours after $\mathrm{CM}$ injection. ${ }^{(6)}$ CAR consensus recommends a similar rate of $1.0 \mathrm{~mL} / \mathrm{kg} / \mathrm{hr}$ but for a longer duration of 12 hours pre- and postprocedure. ${ }^{(3)}$ ACR manuals, on the other hand, recommend a 
Table II. Simplified preventive measures to contrast-induced nephrology in computed tomography.

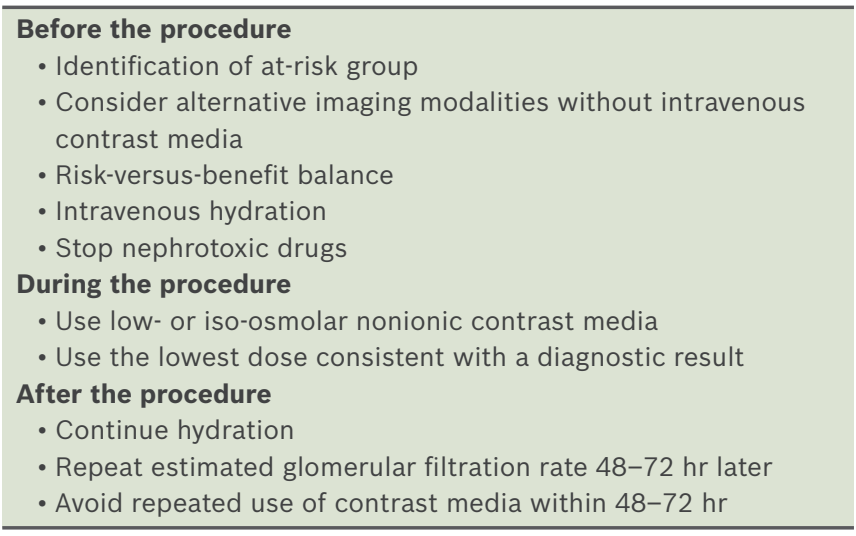

higher infusion rate of $100 \mathrm{~mL} / \mathrm{hr}$ for 6-12 hours before and 4-12 hours after CM injection, ${ }^{(19)}$ while RCR guidelines do not commit on the infusion rate and interval of hydration. ${ }^{(23)}$

\section{Type of fluid regime: $1.4 \%$ of sodium bicarbonate vs $0.9 \%$ of normal saline}

There is ongoing debate on the use of $1.4 \%$ of sodium bicarbonate in $\mathrm{CIN}$ risk reduction. $154 \mathrm{mEq} / \mathrm{L}$ of sodium bicarbonate diluted in dextrose $5 \%$ water is an alternative fluid protocol. ESUR guidelines suggest $3 \mathrm{~mL} / \mathrm{kg} / \mathrm{hr}$ of $1.4 \%$ sodium bicarbonate for 1 hour before and $1 \mathrm{~mL} / \mathrm{kg} / \mathrm{hr}$ for 6 hours after CM injection. This aims to enhance the alkalisation of renal tubular fluid and suppress production of ROS. ${ }^{(36)}$ Merten et al, ${ }^{(37)}$ who published the first randomised controlled trial (RCT) on this subject, described 119 patients who were randomly assigned to receive either $\mathrm{NaCl}$ $154 \mathrm{mEq} / \mathrm{L}$ in $5 \%$ dextrose $/ \mathrm{H}_{2} \mathrm{O}$ or $\mathrm{NaHCO}_{3} 154 \mathrm{mEq} / \mathrm{L}$ in $5 \%$ dextrose $/ \mathrm{H}_{2} \mathrm{O}$. The study showed convincing evidence in favour of $\mathrm{NaHCO}_{3}$ hydration, with $1.7 \%$ of $\mathrm{CIN}$ incidence in the $\mathrm{NaHCO}_{3}$ group compared to $13.6 \%$ in the $\mathrm{NaCl}$ group. ${ }^{(37)}$

Two subsequent RCTs by Recio-Mayoral et al in 2007 and Pakfetrat et al in 2009 compared the effect of a single bolus of $\mathrm{NaHCO}_{3}$ before coronary angiography or $\mathrm{PCl}$; both studies showed a significant reduction of $\mathrm{CIN}$ in the group treated with the $\mathrm{NaHCO}_{3}$ bolus. ${ }^{(38,39)}$ One meta-analysis involving a total of 1,734 patients showed that $\mathrm{NaHCO}_{3}$ is superior to $\mathrm{NaCl}$ alone in the prevention of $\mathrm{CIN}$ in patients with moderate to severe CKD. ${ }^{(40)}$ However, a study of 353 patients undergoing coronary angiography (MEENA trial) showed no benefit of sodium bicarbonate over normal saline in preventing $\mathrm{CIN}{ }^{(41}$ ACR guidelines also state that sodium bicarbonate cannot be considered definitive at this period of time, as it is challenged by a meta-analysis conducted by Zoungas et al in 2009. ${ }^{(42)}$

\section{Route of hydration: oral vs. intravenous}

Intravenous fluid is the preferred route of hydration due to better control of volume expansion. ${ }^{(7)}$ According to ESUR, oral fluid of 1,000 $\mathrm{mL}$ over $6-8$ hours before and after contrast exposure could be sufficient for patients with eGFR of $30-45 \mathrm{~mL} / \mathrm{min}$ and receiving intravenous $\mathrm{CM}$ of $\leq 100 \mathrm{~mL}$. ${ }^{(43)} \mathrm{CAR}$ consensus states that oral hydration is not an evidence-based substitute for intravenous hydration, although some institutions might recommend it in certain outpatients due to the impracticality of intravenous hydration. ${ }^{(3)}$ It was mentioned in the ACR manuals that oral hydration has been utilised but with less demonstrated effectiveness. ${ }^{(19)}$

\section{$\mathrm{N}$-acetylcysteine: is it useful?}

$\mathrm{N}$-acetylcysteine (NAC) was once widely advocated in patients at risk of $\mathrm{CIN}$ following an initial publication by Tepel et al in the year 2000. ${ }^{(44)}$ The typical regime consists of $600 \mathrm{mg}$ of NAC administered orally for two days prior to the procedure. ${ }^{(44)}$ Increasing evidence, however, suggests that NAC is not efficacious in CIN prevention. ${ }^{(45,46)}$ The most meticulous meta-analysis, conducted by Gonzales et al in 2007, also found no beneficial role of NAC in CIN risk reduction. ${ }^{(47)}$ However, based on its ease of use and lack of side effects, many institutions may still opt to add it to a renal protection protocol. ${ }^{(3,43)}$ However, this should not be considered a substitute for hydration. . $^{(3,43)}$

\section{Other drugs}

Many other drugs have also been postulated to play a role in $\mathrm{CIN}$ prophylaxis. These include furosemide, mannitol, fenoldopam, dopamine, atrial natriuretic peptide, calcium channel blocker, L-arginine, endothelin receptor blocker and prostaglandin E1. ${ }^{(48-51)}$ However, the available evidence for the use of these drugs is not convincing, ${ }^{(49)}$ and some have even resulted in harmful side effects to the patients. ${ }^{(49)}$ Potential drugs like theophylline or aminophylline, statins, ascorbic acid, iloprost and nitrates may still require further evaluation. ${ }^{(52-54)}$ Both the ESUR and ACR guidelines opine that renal vasodilators, receptor antagonists of endogenous vasoactive mediators and cytoprotective agents have no consistent protective role against $\mathrm{CIN}$; hence, these agents are not recommended. ${ }^{(6,19)}$

\section{During the procedure}

To reduce the risk of $\mathrm{CIN}$, the majority of the guidelines unanimously recommend the use of low-osmolar CM (e.g. iohexol; Omnipaque $^{\circledR}$ ) or iso-osmolar nonionic CM (e.g. iodixanol; Visipaque ${ }^{\circledR}$ ), with the lowest dose consistent with a diagnostic result. ${ }^{(3,6,19,23)}$ Nevertheless, the available evidence fails to establish a clear advantage of intravenous iso-osmolar iodixanol over intravenous low-osmolar CM with regard to $\mathrm{CIN}{ }^{(55)}$

In general, patients with eGFR $<60 \mathrm{~mL} / \mathrm{min}$ should preferably receive an amount of $\mathrm{CM}$ of not more than $100 \mathrm{~mL}$ in volume. ${ }^{(56)}$ A volume limit of $5 \mathrm{~mL} / \mathrm{kg}$ of body weight normalised to the concentration of serum creatinine has been proposed as the threshold of CIN in CKD patients. ${ }^{(57)} \mathrm{A}$ study by Laskey et al suggests that a $\mathrm{CM}$ volume to creatinine clearance $(\mathrm{CrCl})$ ratio of 3.7 can prospectively determine the maximum volume of $\mathrm{CM}$ without substantially increasing the risk of $\mathrm{CIN}$; a higher risk is seen in patients receiving $\mathrm{CM}$ greater than the ratio of 3.7..$^{(58)}$ A most recent meta-analysis has also proven that reduction of $\mathrm{CM}$ volume with the use of automated contrast injector significantly reduces the incidence of $\mathrm{CIN}$ in patients undergoing coronary angiography. ${ }^{(59)}$ However, according to ACR manuals, there is 
a lack of robust data to support a dose-toxicity relationship for intravenous iodinated $\mathrm{CM}$, as only intracardiac iodinated CM shows a directly proportional relationship. ${ }^{(19)}$

MR imaging is an alternative imaging modality when CT cannot be performed. However, when intravascular CM needs to be administered, none of the international guidelines suggested replacing CT with MR imaging, since gadolinium is also contraindicated in renal-impaired patients. ${ }^{(60)}$ In patients with eGFR $<30 \mathrm{~mL} / \mathrm{min}$, gadolinium carries the risk of a rare and potentially fatal disease known as nephrogenic systemic fibrosis (NSF). ${ }^{(61)}$ According to experimental studies, this disease is due to an activation of circulating fibroblasts following cytokines released by skin macrophages and peripheral blood monocytes. ${ }^{(62)}$ The exact pathogenesis of NSF is unknown. However, it has been postulated that NSF is possibly a result of gadolinium released from its chelates, which subsequently binds to phosphate, causing deposition in the skin and subcutaneous tissues. ${ }^{(62)}$

In general, those with eGFR $<30 \mathrm{~mL} / \mathrm{min}$ are advised to avoid both iodinated intravenous $\mathrm{CM}$ and intravenous gadolinium to conserve the residual renal function and reduce the risk of NSF, respectively. ${ }^{(6,19)}$ Recommendations include alternative imaging procedures or imaging without the use of intravenous CM. Fortunately, with the introduction of macrocyclic gadolinium chelate agents, the risk of NSF has now been largely reduced. This is attributed to the very high complex stability of macrocyclic agents, which decreases the risk of gadolinium ions released in vivo. ${ }^{(63)}$ Some examples of macrocylic agents include gadoterate meglumine (Dotarem), gadobutrol (Gadovist) and gadoteridol (Prohance). ${ }^{(19)}$ After weighing the risks and benefits, if intravenous gadolinium is still deemed absolutely necessary, avoidance of category one gadolinium (e.g. gadodiamide [Omniscan], gadopentetate dimeglumine [Magnevist], gadoversetamide [Optimark]) is advised. ${ }^{(19)}$

\section{After the procedure}

Management of CIN risk does not stop at the completion of contrast-enhanced studies. Volume expansion therapy should continue and eGFR values at 48-72 hours after the procedure should be obtained. These are clearly outlined in the ESUR guidelines. ${ }^{(6)}$ According to the CAR guidelines, repeated use of CM should be avoided within 48-72 hours after the first administration, as a significantly increased risk of $\mathrm{CIN}$ has been demonstrated among patients who received a second dose of CM within 48 hours. ${ }^{(3,64)}$ ACR manuals do not object to the practice of avoiding repeated CM injection within 24 hours. ${ }^{(19)}$

\section{ROLE OF PROPHYLACTIC DIALYSIS OR HAEMOFILTRATION IN CIN}

According to the CAR consensus, dialysis does not play a prophylactic role in reducing the risk of $\mathrm{CIN} .{ }^{(3)}$ Administered CM reaches the kidneys within 1-2 cardiac cycles, making it biologically implausible for removal by dialysis. ${ }^{(3)}$ It is also unlikely to halt the initiated cascade of renal injury when CM has reached the kidneys. ${ }^{(3)}$ The ESUR guidelines do not discuss the prophylactic role of dialysis in patients receiving intravascular $\mathrm{CM}$, likely due to the abovementioned reason. ${ }^{(6)}$ However, it clearly points out that there is no evidence to support the protective role of haemodialysis in $\mathrm{CIN}{ }^{(6)} \mathrm{A}$ meta-analysis by Cruz et al in 2006, which included six RCTs and two non-RCTs, demonstrated no additional benefit of peri-procedural extracorporeal blood purification compared to the standard medical therapy. ${ }^{(64)}$

\section{CIN IN PATIENTS ALREADY ON DIALYSIS}

It has been well established that dialysis patients are not contraindicated to intravenous iodinated CM administration. ${ }^{(6,19)}$ ACR guidelines state that intravenous CM administration has a theoretical risk of causing a dialysis patient to change from an oliguric state to an anuric state, but this remains speculative, with no conclusive data seen. ${ }^{(19)}$ Dialysis patients are also at a theoretical risk of osmotic load from intravenous iodinated CM, as they are unable to remove the imposed excessive intravascular volume. ${ }^{(19)}$ The CM dosing should therefore be as low as necessary. ${ }^{(19)}$ There is no need for an urgent dialysis unless an unusually large volume of CM is used, or there is a substantial underlying cardiac dysfunction. ${ }^{(65)}$ ESUR guidelines also state that it is not necessary to correlate the time of CM injection with haemodialysis sessions, or to have extra haemodialysis sessions. ${ }^{(6)}$

\section{CONCLUSION}

Despite the numerous retrospective studies published, the exact pathogenesis of renal-related adverse effects of intravascular $\mathrm{CM}$ has yet to be completely understood. Nevertheless, every healthcare personnel should be aware of CIN, as at-risk patients have become increasingly more common. While the exact pathogenesis of CIN is unknown, interventions must be put in place. In patients receiving intravenous iodinated $\mathrm{CM}$, the previously accepted threshold of eGFR $<60 \mathrm{~mL} / \mathrm{min}$ has been lowered to eGFR $<45 \mathrm{~mL} / \mathrm{min}$, while a threshold of $\mathrm{eGFR}<60 \mathrm{~mL} / \mathrm{min}$ remains for $\mathrm{CM}$ delivered via the intra-arterial route.

To reduce the risk of $\mathrm{CIN}$, hydration remains the mainstay of $\mathrm{CIN}$ prophylaxis in at-risk groups, and should continue into the post-procedural period. The benefits of sodium bicarbonate and NAC have been put to question in recent meta-analyses. Overall, the use of intravascular CM should be clinically justified and balanced between the risks and benefits of use, with consideration of alternative imaging modalities.

\section{REFERENCES}

1. Schlifke A, Geiderman JM. Seafood allergy is a specific and unique contraindication to the administration of ionic contrast media. CJEM 2003; 5:166-8.

2. McCullough PA. Contrast-induced acute kidney injury. J Am Coll Cardiol 2008; 51:1419-28.

3. Owen RJ, Hiremath S, Myers A, Fraser-Hill M, Barrett B. Consensus Guidelines for the Prevention of Contrast Induced Nephropathy. Available at: http://www.car.ca/uploads/standards\%20guidelines/20110617_en_ prevention_cin.pdf. Accessed December 23, 2013.

4. Ad-hoc working group of ERBP, Fliser D, Laville M, et al. A European Renal Best Practice (ERBP) position statement on the Kidney Disease Improving Global Outcomes (KDIGO) clinical practice guidelines on acute kidney injury: part 1: definitions, conservative management and contrast-induced nephropathy. Nephrol Dial Transplant 2012; 27:4263-72. 
5. Bellomo R, Kellum JA, Ronco C. Acute kidney injury. Lancet 2012; 380:756-66.

6. European Society of Urogenital Radiology Guidelines on Contrast Media In: European Society of Urogenital Radiology [online]. Available at: http:// www.esur.org/guidelines/. Accessed December 23, 2013.

7. Geenen RW, Kingma HJ, van der Molen AJ. Contrast-induced nephropathy: pharmacology, pathophysiology and prevention. Insights Imaging 2013 4:811-20.

8. Chong E, Shen L, Poh KK, Tan HC. Risk scoring system for prediction of contrast-induced nephropathy in patients with pre-existing rena impairment undergoing percutaneous coronary intervention. Singapore Med J 2012; 53:164-9.

9. Tsai TT, Patel UD, Chang TI, et al. Contemporary incidence, predictors and outcomes of acute kidney injury in patients undergoing percutaneous coronary interventions: insights from the NCDR Cath- $\mathrm{PCl}$ registry. JACC Cardiovasc Interv 2014; 7:1-9.

10. Neyra JA, Shah S, Mooney R, et al. Contrast-induced acute kidney injury following coronary angiography: a cohort study of hospitalized patients with or without chronic kidney disease. Nephrol Dial Transplant 2013 28:1463-71.

11. Go AS, Chertow GM, Fan D, McCulloch CE, Hsu CY. Chronic kidney disease and the risks of death, cardiovascular events, and hospitalization. N Engl J Med 2004; 351:1296-305.

12. Thomsen HS, Morcos SK. Contrast media and the kidney: European Society of Urogenital Radiology (ESUR) guidelines. Br J Radiol 2003; 76:513-8.

13. Brezis M, Rosen S. Hypoxia of the renal medulla--its implications for disease. N Engl J Med 1995; 332:647-55.

14. Heyman SN, Rosen S, Rosenberger C. Renal parenchymal hypoxia, hypoxia adaptation, and the pathogenesis of radiocontrast nephropathy. Clin J Am Soc Nephrol 2008; 3:288-96.

15. Katzberg RW. Contrast medium-induced nephrotoxicity: which pathway? Radiology 2005; 235:752-5.

16. Persson PB, Hansell P, Liss P. Pathophysiology of contrast medium-induced nephropathy. Kidney Int 2005; 68:14-22.

17. Heyman SN, Rosen S, Khamaisi M, Idée JM, Rosenberger C. Reactive oxygen species and the pathogenesis of radiocontrast-induced nephropathy. Invest Radiol 2010; 45:188-95.

18. Haller C, Hizoh I. The cytotoxicity of iodinated radiocontrast agents on renal cells in vitro. Invest Radiol 2004; 39:149-54.

19. ACR Committee on Drugs and Contrast Media. ACR Manual on Contrast Media Version 9. In: American College of Radiology [online]. Available at: http://www.acr.org/ /media/ACR/Documents/PDF/QualitySafety/ Resources/Contrast\%20Manual/2013_Contrast_Media.pdf. Accessed December 23, 2013.

20. Rudnick MR, Goldfarb S, Wexler L, et al. Nephrotoxicity of ionic and nonionic contrast media in 1196 patients: a randomized trial. The lohexo Cooperative Study. Kidney Int 1995; 47:254-61.

21. Moore RD, Steinberg EP, Powe NR, et al. Nephrotoxicity of high-osmolality versus low-osmolality contrast media: randomized clinical trial. Radiology 1992; 182:649-55.

22. Katzberg RW, Barrett BJ. Risk of iodinated contrast material--induced nephropathy with intravenous administration. Radiology 2007; 243:622-8.

23. Standards for intravascular contrast agent administration to adult patients. 2nd ed. In: The Royal College of Radiologists [online]. Available at http://www.rcr.ac.uk/docs/radiology/pdf/BFCR(10) 4_Stand_contrast.pdf. Accessed December 23, 2013.

24. Katzberg RW, Newhouse JH. Intravenous contrast medium-induced nephrotoxicity: is the medical risk really as great as we have come to believe? Radiology 2010; 256:21-8

25. Mehran R, Aymong ED, Nikolsky E, et al. A simple risk score for prediction of contrast-induced nephropathy after percutaneous coronary intervention: development and initial validation. J Am Coll Cardiol 2004; 44:1393-9.

26. Okino S, Fukuzawa S, Inagaki M, et al. Hyperuricemia as a risk factor for progressive renal insufficiency after coronary intervention in patients with chronic kidney disease. Cardiovasc Interv Ther 2010; 25:105-11.

27. Saritemur M, Turkeli M, Kalkan K, Tanboga İH, Aksakal E. Relation of uric acid and contrast-induced nephropathy in patients undergoing primary percutaneous coronary intervention in the ED. Am J Emerg Med 2014; 32:119-23.

28. Cochran ST. Determination of serum creatinine level prior to administration of radiographic contrast media. JAMA 1997; 277:517-8.

29. Tippins RB, Torres WE, Baumgartner BR, Baumgarten DA. Are screening serum creatinine levels necessary prior to outpatient $C T$ examinations? Radiology 2000; 216:481-4.

30. Choyke PL, Cady J, DePollar SL, Austin H. Determination of serum creatinine prior to iodinated contrast media: is it necessary in all patients? Tech Urol 1998; 4:65-9.

31. Glassock RJ, Winearls C. Screening for CKD with eGFR: doubts and dangers. Clin J Am Soc Nephrol 2008; 3:1563-8.

32. Ellis JH, Cohan RH. Reducing the risk of contrast-induced nephropathy: a perspective on the controversies. AJR Am J Roentgenol 2009; 192:1544-9.

33. Pires de Freitas do Carmo L, Macedo E. Contrast-induced nephropathy: attributable incidence and potential harm. Crit Care 2012; 16:127.

34. Weisbord SD, Palevsky PM. Prevention of contrast-induced nephropathy with volume expansion. Clin J Am Soc Nephrol 2008; 3:273-80.

35. Mueller C, Buerkle G, Buettner HJ, et al. Prevention of contrast mediaassociated nephropathy: randomized comparison of 2 hydration regimens in 1620 patients undergoing coronary angioplasty. Arch Intern Med 2002; 162:329-36.

36. Ellis JH, Cohan RH. Prevention of contrast-induced nephropathy: an overview. Radiol Clin North Am 2009; 47:801-11

37. Merten GJ, Burgess PW, Gray LV, et al. Prevention of contrast-induced nephropathy with sodium bicarbonate: a randomized controlled trial. JAMA 2004; 291:2328-34

38. Recio-Mayoral A, Chaparro M, Prado B, et al. The reno-protective effect of hydration with sodium bicarbonate plus $\mathrm{N}$-acetylcysteine in patients undergoing emergency percutaneous coronary intervention: the RENO study. J Am Coll Cardiol 2007; 49:1283-8.

39. Pakfetrat M, Nikoo MH, Malekmakan L, et al. A comparison of sodium bicarbonate infusion versus normal saline infusion and its combination with oral acetezolamide for prevention of contrast-induced nephropathy: a randomized, double-blind trial. Int Urol Nephrol 2009; 41:629-34.

40. Kunadian V, Zaman A, Spyridopoulos I, Qiu W. Sodium bicarbonate for the prevention of contrast induced nephropathy: a meta-analysis of published clinical trials. Eur J Radiol 2011; 79:48-55.

41. Brar SS, Shen AY, Jorgensen MB, et al. Sodium bicarbonate vs sodium chloride for the prevention of contrast medium-induced nephropathy in patients undergoing coronary angiography: a randomized trial. JAMA 2008; 300:1038-46

42. Zoungas S, Ninomiya T, Huxley $R$, et al. Systematic review: sodium bicarbonate treatment regimens for the prevention of contrast-induced nephropathy. Ann Intern Med 2009; 151:631-8.

43. Teaching Module V. Contrast-Induced Nephropathy - CIN. In: European Society of Urogenital Radiology [online]. Available at: http://www.esur. org/fileadmin/content/Contrast_Media_Education/N_2011_CIN_Module. pdf. Accessed December 23, 2013.

44. Tepel M, van der Giet M, Schwarzfeld C, et al. Prevention of radiographiccontrast-agent-induced reductions in renal function by acetylcysteine. N Engl J Med 2000; 343:180-4.

45. Marenzi G, Assanelli E, Marana I, et al. N-acetylcysteine and contrastinduced nephropathy in primary angioplasty. N Engl J Med 2006; 354:2773-82

46. Thiele H, Hildebrand L, Schirdewahn C, et al. Impact of high-dose $\mathrm{N}$-acetylcysteine versus placebo on contrast-induced nephropathy and myocardial reperfusion injury in unselected patients with ST-segment elevation myocardial infarction undergoing primary percutaneous coronary intervention. The LIPSIA-N-ACC (Prospective, Single-Blind, PlaceboControlled, Randomized Leipzig Immediate PercutaneouS Coronary Intervention Acute Myocardial Infarction N-ACC) Trial. J Am Coll Cardiol 2010; 55:2201-9.

47. Gonzales DA, Norsworthy KJ, Kern SJ, et al. A meta-analysis of $\mathrm{N}$-acetylcysteine in contrast-induced nephrotoxicity: unsupervised clustering to resolve heterogeneity. BMC Med 2007; 5:32.

48. Briguori C, Colombo A, Airoldi F, et al. N-Acetylcysteine versus fenoldopam mesylate to prevent contrast agent-associated nephrotoxicity. J Am Coll Cardiol 2004; 44:762-5

49. Majumdar SR, Kjellstrand CM, Tymchak WJ, et al. Forced euvolemic diuresis with mannitol and furosemide for prevention of contrast-induced nephropathy in patients with CKD undergoing coronary angiography: a randomized controlled trial. Am J Kidney Dis 2009; 54:602-9.

50. Ng TM, Shurmur SW, Silver M, et al. Comparison of $\mathrm{N}$-acetylcysteine and fenoldopam for preventing contrast-induced nephropathy (CAFCIN). Int J Cardiol 2006; 109:322-8.

51. Stone GW, McCullough PA, Tumlin JA, et al; CONTRAST Investigators. Fenoldopam mesylate for the prevention of contrast-induced nephropathy: a randomized controlled trial. JAMA 2003; 290:2284-91.

52. Boscheri A, Weinbrenner C, Botzek B, et al. Failure of ascorbic acid to prevent contrast-media induced nephropathy in patients with renal dysfunction. Clin Nephrol 2007; 68:279-86.

53. Kwok CS, Pang CL, Yeong JK, Loke YK. Measures used to treat 
contrast-induced nephropathy: overview of reviews. Br J Radiol 2013; $86: 20120272$.

54. Peguero JG, Cornielle V, Gomez SI, et al. The use of nitrates in the prevention of contrast-induced nephropathy in patients hospitalized after undergoing percutaneous coronary intervention. J Cardiovasc Pharmacol Ther 2014; 19:310-4

55. From AM, Al Badarin FJ, McDonald FS, et al. lodixanol versus low-osmolar contrast media for prevention of contrast nephropathy: meta-analysis of randomized, controlled trials. Circ Cardiovasc Interv 2010; 3:351-8.

56. Davidson CJ, Hlatky M, Morris KG, et al. Cardiovascular and renal toxicity of a nonionic radiographic contrast agent after cardiac catheterization. A prospective trial. Ann Intern Med 1989; 110:119-24.

57. Cigarroa RG, Lange RA, Williams RH, Hillis LD. Dosing of contrast materia to prevent contrast nephropathy in patients with renal disease. Am J Med 1989; 86(6 Pt 1):649-52.

58. Laskey WK, Jenkins C, Selzer F, et al; NHLBI Dynamic Registry Investigators Volume-to-creatinine clearance ratio: a pharmacokinetically based risk factor for prediction of early creatinine increase after percutaneous coronary intervention. J Am Coll Cardiol 2007; 50:584-90.

59. Minsinger KD, Kassis HM, Block CA, Sidhu M, Brown JR. Meta-analysis of the effect of automated contrast injection devices versus manual injection and contrast volume on risk of contrast-induced nephropathy. Am J Cardiol 2014; 113:49-53

60. Boyden TF, Gurm HS. Does gadolinium-based angiography protect against contrast-induced nephropathy?: a systematic review of the literature. Catheter Cardiovasc Interv 2008; 71:687-93.

61. Cowper SE. Nephrogenic systemic fibrosis: an overview. J Am Coll Radiol 2008; 5:23-8.

62. Chopra T, Kandukurti K, Shah S, Ahmed R, Panesar M. Understanding nephrogenic systemic fibrosis. Int I Nephrol 2012; 2012:912189.

63. Seeger A, Kramer U, Fenchel M, et al. Comparison between a linear versus a macrocyclic contrast agent for whole body MR angiography in a clinical routine setting. J Cardiovasc Magn Reson 2008; 10:63.

64. Cruz DN, Perazella MA, Bellomo R, et al. Extracorporeal blood purification therapies for prevention of radiocontrast-induced nephropathy: a systematic review. Am J Kidney Dis 2006; 48:361-71.

65. Younathan CM, Kaude JV, Cook MD, Shaw GS, Peterson JC. Dialysis is not indicated immediately after administration of nonionic contrast agents in patients with end-stage renal disease treated by maintenance dialysis. AJR Am J Roentgenol 1994; 163:969-71. 